\title{
Validades existenciais e enigmas relacionados
}

Paulo A. S. Veloso

UFRJssmv@bridge.com.br

Luiz Carlos Pereira

PUC-RJ/UFRJ1luiz@inf.puc-rio.br

Edward H. Haeusler

PUC-RJ

resumo A lógica não contém teoremas puramente existenciais: as únicas sentenças existenciais válidas são aquelas com análogas universais válidas. Aqui, mostramos que isto realmente é assim quando corretamente interpretado: toda validade ex- istencial possui uma análoga universal simples, que também é válida. Também caracterizamos validades universais e existenciais em termos de tautologias.

palavras-chave Lógica; quantificação; validades existenciais; validades universais; tautologias

\section{Introdução}

A lógica não deve conter teoremas puramente existenciais: as únicas sentenças existenciais válidas são aquelas com análogas universais válidas. Frequentemente ouve-se este chavão, bem como, às vezes, algumas possíveis explicações para ele ${ }^{1}$

Um esboço de explicação intuitiva para este chavão poderia ser o seguinte. Considere uma assertiva existencial como "existem pintalouvas" ou "algo é uma pintalouva". Tais assertivas podem talvez ser derivadas de algumas hipóteses. Mas, como poderiam ser provadas, a partir de nenhuma hipótese? Neste contexto, não se tem a mínima idéia do que "pintalouva" supostamente significa ${ }^{2}$. Por isso, pode-se apelar apenas a princípios lógicos. Como seria uma tal prova?

Além disso, quando acontece de "algo é uma pintalouva" ser verdadeiro, percebe-se uma certa assimetria, distinguindo pintalouvas de não-pintalouvas. Novamente, isso pode ser bastante razoável se tivermos Recebido em 7 de março de 2009. Aceito em 21 de agosto de 2009. doispontos, Curitiba, São Carlos, vol. 6, n. 2, p.145-163, outubro, 2009 


\section{6}

alguma informação sobre estes conceitos. Mas, pode isto ser verdade apenas com base na lógica? Sente-se que a assimetria acima vai contra o espírito lógico: ela seria quebrada caso tudo fosse uma pintalouva.

Aqui, mostramos que toda validade existencial possui uma análoga universal simples que também é válida. Também caracterizamos as validades existenciais e universais em termos de tautologias: mostramos como transformar sentenças existenciais e universais em suas contrapartidas proposicionais, que serão tautológicas exatamente quando as sentenças originais forem válidas.

Uma versão do chavão anterior é que a lógica não tem compromissos existenciais. Bem, em um certo sentido, ela tem. Comumente supõe-se que símbolos para constantes $\mathrm{x}$ denotam elementos do universo; assim a sentença existencial $\exists x x=c$ é válida ${ }^{3}$. Similarmente, símbolos para funções representam funções totais no universo; assim a sentença existencial $\exists x \exists y y=f(x)$ é válida ${ }^{4}$. Na ausência de símbolos para constantes ou funções, que é o caso que examinaremos aqui, não se tem tais compromissos existenciais. Talvez se possa detectar um outro compromisso existencial no fato de que a lógica considera apenas universos não-vazios, o que torna válida a sentença existencial $\exists x x=x$; não excluiremos tais casos ${ }^{5}$.

A estrutura destre trabalho é a seguinte. $\mathrm{Na}$ seção 2, introduziremos informalmente nossos conceitos, métodos e resultados. Na seção 3, demonstraremos conexões entre sentenças existenciais e universais. $\mathrm{Na}$ seção 3, estabeleceremos reduções proposicionais para sentenças existenciais e universais. $\mathrm{Na}$ seção 5, faremos um restrospecto de nossos conceitos e resultados principais. Na seção 6, concluiremos com comentários sobre algumas possíveis extensões de nosso enfoque, colocando-os em contexto.

\section{Ideias Básicas}

Nesta seção, vamos introduzir informalmente, enfatizando exemplos, alguns conceitos, métodos e resultados que serão desenvolvidos a seguir: nas seções 3 e 4 . Consideraremos uma linguagem de primeira ordem sem símbolos para constantes ou funções. 


\subsection{Validades existênciais e versões universais}

Começaremos com alguns exemplos bem simples.

A sentença cxistencial $\rfloor x x \quad x$ ć válida, c também é válida sua versão universal $\vee x x=x$. Além disso, dado um (símbolo de) predicado unário $p$, a sentença existencial $\exists x p(x)$ não é válida, nem tampouco é válida a sua versão universal $\vee x p(x)$.

Em contraste, a sentença existencial $\exists x \exists y x=y$ é válida, ao passo que sua versão universal $\forall x \forall y x=y$ não é válida. Note, no entanto, que $\exists x \exists y x=y$ é logicamente equivalente a $\exists x \exists y(x=y \vee x=x)$ e a versão universal desta, $\forall x \forall y(x=y \vee x=x)$, também é válida. Aqui aplicamos uma transformação puramente sintática.

A sentença existencial $\exists x \exists y r(x, y)$ será transformada em $\exists x \exists y[r(x, y) \vee r(x, x)]$. Note que em $\exists x \exists y[r(x, y) \vee r(x, x)]$, a parte $r(x, x)$ "internaliza" a alternativa de satisfação de $r(x, y)$ por um par com componentes idênticos. No caso de uma única variável, passamos de $\sqsupset x q(x)$ a $\sqsupset x|q(x) \vee q(x)|$.

Tais transformações sintáticas podem ser feitas em duas ctapas. Considere uma fórmula $M(x, y, z)$ c sclecione uma de suas variáveis, digamos $y$. Inicialmente, construímos sua instância singular (cm $y$ ) substituindo cada ocorrência das variáveis $x, y, z$ por $y$, obtendo a fórmula $M(y, y, y)$. Em seguida, construa a sua versão alternativa (em $y$ ) como a disjunção $M(x, y, z) \vee M(y, y, y)$.

Agora, se $x, y, z$ são as únicas variáveis ocorrendo livres em $M(x, y, z)$, temos uma sentença $\exists x \exists y \exists z M(x, y, z)$. Fissa sentença é logicamente equivalente a sua versão alternativa $\exists x \exists y \exists z,[M(x, y, z) \vee M(y, y, y)]$.

Como estamos interessados em saber se a sentença existencial como $\exists x \exists y \exists z M(x, y, z)$ é válida ou não, podemos substituí-la por qualquer outra equivalente, em particular pela obtida por nossa transformação sintática simples: $\sqsupset x \sqsupset y \sqsupset z|M(x, y, z) \vee M(y, y, y)|$.

Alćm disso, quando a fórmula $M(x, y, z)$ é uma matriz, i.c. sem quantificadores, as sentenças

$$
\exists x \exists y \exists z M(x, y, z) \mathrm{e} \exists x \exists y \exists z[M(x, y, z) \vee M(y, y, y)]
$$

são puramente existenciais. Nesse caso, a sentença existencial alternativa

$$
\exists x \exists y \sqsupset z[M(x, y, z) \vee M(y, y, y)]
$$

é válida sce, c somente sce é válida a sua versão universal

$$
\forall x \forall y \forall z[M(x, y, z) \vee M(y, y, y)]
$$

Essa última afirmativa pode parecer estranha, principalmente se lembramos que a sentença existencial $\exists x \exists y \exists z M(x, y, z)$ é válida se, e somente se, ela é satisfeita em toda estrutura com um único elemento [vl)a89, p. 93, exercício 11]. Note, contudo, que a versão universal tem uma matriz alternativa,

A versão alternativa é bastante peculiar. lara uma sentença em forma prenex

$$
\forall x \exists y \forall z[M(x, y, z) \vee M(y, y, y)] \text {, }
$$

os quantificadores no prefixo não exercem nenhum papel na determinação de se cla é válida ou não. Pelo resultado acima, vê-se que são equivalentes as assertivas seguintes.

1. A sentença prenex $\forall x \exists y \forall z[M(x, y, z) \vee M(y, y, y)]$ é válida.

2. A sentença universal $\vee x \vee y \vee z[M(x, y, z) \vee M(y, y, y)]$ é válida. 
3. A sentença existencial $\sqsupset x \sqsupset y \sqsupset z|M(x, y, z) \vee M(y, y, y)|$ é válida.

Além disso, quando sentença prenex $\forall x \exists y \forall z$. $[M(x, y, z) \vee M(y, y, y)]$ é válida, também é válida a versão singular $M(y, y, y)$ de sua matriz.

Essas idéias serão desenvolvidas com mais cuidado na seção 3 .

2.2 Validades existenciais e tautologias

As idéias acima levam à seguinte tentativa de se oferecer uma explicação mais intuitiva: em uma estrutura com um único elemento, não há como distinguir os quantificadores $\exists$ e $\forall$. Assim, deveríamos ter explicações puramente proposicionals ${ }^{6}$.

Por exemplo, considere as sentenças existenciais

$$
\exists x \exists y[r(x, y) \rightarrow r(y, x)] \text { e } \exists x \exists y[r(x, y) \vee r(y, x)] .
$$

Apague os quantificadores c substitua cada variável por uma única variável $v^{7}$. Então,

1. a primeira se torna $[r(v, v) \rightarrow r(v, v)]$ (uma tautologia),

2. enquanto a segunda se torna $[r(v, v) \vee r(v, v)]$ (que não é uma tautologia)

Obtém-se a versão proposicional singular $F_{v}$ de uma fórmula $F$ apagando-se todos os seus quantificadores e substituindo cada parte atômica $p\left(v_{1}, \cdots, v_{n}\right)$ por $p(v, \cdots, v)$ e $u=v$ por $\mid$ (verdadeiro).

Iessa mancira, uma sentença puramente existencial $\sqsupset v_{1} \ldots J v_{n} M$ é válida se, e somente se, a sua versão proposicional singular $M_{v}$ é uma tautologia. Essas idéias scrão desenvolvidas em mais detalhes adiantc (cᄃ. 4.1).

2.3 Validades existenciais e universais como tautologias

A situação parece ser a seguinte:

- Por um lado, cada sentença universal $\vee x \vee y \vee z, M(x, y, z)$ que é válida dá origem a uma sentença existencial $\exists x \exists y \exists z M(x, y, z)$ que também é válida.

- Por outro lado, uma sentença puramente existencial válida na forma alternativa

$$
\exists x \exists y \exists z[M(x, y, z) \vee M(y, y, y)]
$$

dá origem à sentença universal

$$
\forall x \forall y \forall z[M(x, y, z) \vee M(y, y, y)],
$$

que também é válida.

Desta forma, poder-se-ia dizer que há “mais" validades existenciais do que universais. Isto parece razoável, mas não poderia haver uma explicação mais proposicional? 
Dada uma matriz $M(x, y, z)$ (sem quantificadores), buscamos condições para a validade de cada uma das seguintes sentenças:

( $)$ scu fecho existencial $\sqsupset x \sqsupset y \sqsupset z M(x, y, z)$,

$(\underline{\forall})$ seu fecho universal $\forall x \forall y \forall z, M(x, y, z)$.

Por excmplo: para a matri\% $|r(x, y) \quad>r(y, x)|$,

- o fecho existencial $\exists x \exists y[r(x, y) \rightarrow r(y, x)]$ é válido (pois $r(v, v) \rightarrow r(v, v)$ é uma tautologia);

- o fecho universal $\forall x \forall y|r(x, y) \quad>r(y, x)|$ não é válido (note que $r(x, y) \quad>r(y, x)$ não é uma tautologia).

Explicação proposicional: condições para validade (para linguagem sem identidade $=$ ).

(c) Para (马): quando a instância singular $M(v, v, v)$ for uma tautologia.

(u) Para $(\underline{\forall})$ : quando a matriz $M(x, y, z)$ for uma tautologia.

Estas idcias proporcionam uma explicação proposicional para a concxão antcrior entre validades existenciais c universais. Suponha que scja válida a sentença existencial

$$
\exists x \exists y \exists z, M(x, y, z) .
$$

Fntão por (e), sua instância singular $M(y, y, y)$ é uma tautologia. Agora, considere a versão alternativa universal

$$
\vee x \vee y \vee z[M(x, y, z) \vee M(y, y, y)] .
$$

Sua matriz é $M(x, y, z) \vee M(y, y, y)$, que também é uma tautologia (por seguir-se da tautologia $M(y, y, y))$. Logo, por (u), a versão universal alternativa também é válida.

Os resultados anunciados nesta seção também valem no contexto de uma teoria universal: um conjunto de sentenças universais.

\section{Validades Existenciais e Universais}

Agora demonstraremos as conexões anunciadas entre sentenças existenciais e universais. Lembre-se que trabalhamos com uma linguagem de primeira ordem $\mathrm{L}$ sem símbolos para constantes ou funções.Usaremos $\models_{\mathrm{L}}$ para consequência lógica (em L).

Começamos precisando alguns conceitos apresentados infomalmente na seção 2.

1. Uma sentença é uma fórmula scm (ocorrências de) variáveis livres.

2. Uma matriz é uma fórmula sem quantificadores.

3. Uma fórmula prenex é da forma $Q_{1} v_{1} \ldots Q_{n} v_{n} M$, onde cada $Q_{i}$ é um quantificador ( $\forall$ ou $\sqsupset$ ) c $M$ ć uma matri $\%$. 
4. Uma fórmula (puramente) existencial é uma fórmula prenex cujos quantificadores são todos existenciais, i. e. da forma $\exists v_{1} \ldots \exists v_{n} M$, sendo $M$ uma matriz.

5. Uma fórmula (puramente) universal é uma fórmula prenex cujos quantificadores são todos universais, i. c. da forma $\forall v_{1} \ldots \forall v_{n} M$, sendo $M$ uma matri\%.

Agora, vamos examinar as transformações sintáticas. Uma tal transformação parece ser indispensável. Pode-se talve\% perecber melhor a necessidade de alguma transformação, por meio de dois resultados simples e bem conhecidos ([vI)a89, p. 93, exercício 11]). Esses resultados, dando condições de validade para sentenças existenciais e universais, podem servir para contrastar validades existenciais e universais. Considerando uma matriz $M\left(v_{1}, \ldots, v_{n}\right)$ (sem quantificadores);

$\left(\varepsilon_{1}\right)$ a sentença (puramente) existencial $\exists v_{1} \ldots \exists v_{n} M\left(v_{1}, \ldots, v_{n}\right)$ é válida se, e somente se, cla vale em toda cstrutura com um único elemento,

$\left(v_{n}\right)$ a sentença (puramente) universal $\forall v_{1} \ldots \forall v_{n} M\left(v_{1}, \ldots, v_{n}\right)$ é válida se, e somente se, ela vale em toda estrutura com, no máximo, $n$ elementos.

As transformações sintálicas são como se segue. Considere uma fórmula $F$ e selecionc uma variável $v$.

1. A instância singular em $v$ de $F$ (que denotaremos por $F_{v}$ ) é o resultado de substituir cada ocorrência livre de variável em $F$ por $v$.

2. A versão alternativa em $v$ de $F$ (que denotaremos por $F_{v}^{\prime}$ ) é construída como a disjunção $F \vee F_{v}$.

3. Quando a variável selecionada $v$ ocorre livre em $F$, diremos que:

(a) a instância singular $F_{v}$ ć uma instância singular de $F$;

(b) a versão alternativa $F_{v}^{\prime}$ ć uma versão alternativa de $F$.

A equivalência entre fechos existenciais e suas versões alternativas é imediata.

Lema 3.1. Dada uma fórmula $F\left(v_{1}, \ldots, v_{k}, \ldots, v_{n}\right)$, cujas variáveis livres são $v_{1}, \ldots, v_{k}, \ldots, v_{n}$, a sentença existencial quantificada

$$
\sqsupset v_{1} \ldots \sqsupset v_{k} \ldots \sqsupset v_{n} F\left(v_{1}, \ldots, v_{k}, \ldots, v_{n}\right)
$$

é logicamente equivalente a cada versão alternativa

$$
\exists v_{1} \ldots \exists v_{k} \ldots \exists v_{n}\left[F\left(v_{1}, \ldots, v_{k}, \ldots, v_{n}\right) \vee F\left(v_{k}, \ldots, v_{k}, \ldots, v_{k}\right)\right] .
$$

Demonstração. A equivalência decorre de regras de eliminação e de introdução.

$(\vdash)$ Por $\vee$-introdução, $\exists$-introduções e $\exists$-eliminações ${ }^{8}$.

$(\dashv)$ P’or Э-introduçōes, $\vee$-eliminação e $\exists$-eliminações 9

Portanto, temos a equivalência entre as duas sentenças.

Agora, temos nosso resultado básico conectando sentenças existenciais e universais que são consequências de uma teoria universal: um conjunto de sentenças universais.

Proposição 3.1. Considere um conjunto $\Gamma$ de sentenças universais. Dada uma matriz, $M\left(v_{1}, \ldots, v_{k}, \ldots, v_{n}\right)$, as seguintes assertivas são equivalentes. 
(ع) A sentença existencial

$$
\exists v_{1} \ldots \exists v_{k} \ldots \exists v_{n} M\left(v_{1}, \ldots, v_{k}, \ldots, v_{n}\right)
$$

é uma consequência de $\Gamma$.

$\left(\varepsilon_{1}\right)$ A sentença existencial

$$
\exists v_{1} \ldots \exists v_{k} \ldots \exists v_{n} M\left(v_{1}, \ldots, v_{k}, \ldots, v_{n}\right)
$$

é satisfeita por todo modelo de l'com um único elemento.

$\left(v^{\prime}\right)$ A sentença universal alternativa

$$
\vee v_{1} \ldots \vee v_{k} \ldots \vee v_{n}\left[M\left(v_{1}, \ldots, v_{k}, \ldots, v_{n} \vee M\left(v_{k}, \ldots, v_{k}, \ldots, v_{k}\right)\right]\right.
$$

é uma consequência de $\Gamma$.

$\left(\varepsilon^{\prime}\right)$ A sentença existencial alternativa

$$
\exists v_{1} \ldots \exists v_{k} \ldots \exists v_{n}\left[M\left(v_{1}, \ldots, v_{k}, \ldots, v_{n} \vee M\left(v_{k}, \ldots, v_{k}, \ldots, v_{k}\right)\right]\right.
$$

é uma consequência de $\Gamma$.

Demonstração. Claramente $(\varepsilon) \Rightarrow\left(\varepsilon_{1}\right)$ e $\left(v^{\prime}\right) \Rightarrow\left(\varepsilon^{\prime}\right)$. O lema 3.1 nos dá $\left(\varepsilon^{\prime}\right) \Rightarrow(\varepsilon)$. Para ver que $\left(\varepsilon_{1}\right) \Rightarrow\left(v^{\prime}\right)$, suponha que não temos $\left(v^{\prime}\right)$. Então, algum modelo $\mathfrak{S}$ de $\mathbb{l}^{\prime}$ não satisfaz a sentença universal alternativa

$$
\vee v_{1} \ldots \vee v_{k} \ldots \vee v_{n}\left[M\left(v_{1}, \ldots, v_{k}, \ldots, v_{n} \vee M\left(v_{k}, \ldots, v_{k}, \ldots, v_{k}\right)\right] .\right.
$$

Logo, $\mathfrak{S}$ satisfara a sentença existencial

$$
\exists v_{1} \ldots \exists v_{k} \ldots \exists v_{n}\left[\quad M\left(v_{1}, \ldots, v_{k}, \ldots, v_{n}\right) \wedge \quad M\left(v_{k}, \ldots, v_{k}, \ldots, v_{k}\right)\right] .
$$

Assim, temos

$$
\mathfrak{S} \mid \sqsupset v_{1} \ldots \sqsupset v_{k} \ldots \sqsupset v_{n} \neg M\left(v_{k}, \ldots, v_{k}, \ldots, v_{k}\right) .
$$

Deste modo, para algum clemento $s \subset S$, temos

$$
\mathfrak{S} \models \neg M\left(v_{k}, \ldots, v_{k}, \ldots, v_{k}\right)[[s] .
$$

Agora, considere a sub-estrutura $\mathfrak{S}^{*} \mathrm{de} \mathfrak{S} \mathrm{c}$ com universo $\{s\}^{10}$. Notc que

1. a estrutura $\mathfrak{S}^{*}$ é um modelo de $\Gamma$ com um único elemento (pois $\Gamma$ consiste de sentenças universais);

2. a sentença $\exists v_{1} \ldots \exists v_{k} \ldots \exists v_{n} M\left(v_{1}, \ldots, v_{k}, \ldots, v_{n}\right)$ não é satisfeita em $\mathfrak{S}^{*}$

( $O$ único candidato possível para os $v_{i}$ 's seria $s$, o que não funciona ${ }^{11}$ ).

Portanto, $\mathfrak{S}^{*}$ é um modelo de $\Gamma$, com um único elemento, que não satisfáa a sentença existencial $\exists v_{1} \ldots \exists v_{k} \ldots \exists v_{n} M\left(v_{1}, \ldots, v_{k}, \ldots, v_{n}\right)$.

Note que a equivalência $(\varepsilon) \Leftrightarrow\left(\varepsilon_{1}\right)$ estabelece a condição de validade para sentenças existenciais mencionada acima. Temos também nossa conexão entre consequências existenciais e universais de uma teoria universal. 
Teorema 3.1. Considere um conjunto $\Gamma$ de sentenças universais. Dada uma matriz $M(\underline{v})$ e uma variável $v$ em $\underline{\underline{v}}$, as seguintes assertivas são equivalentes.

ع) $\Lambda$ sentença existencial $\sqsupseteq \underline{v} M(\underline{v})$ é uma consequência de $\Gamma: \Gamma \mid \mathrm{I} . \underline{\underline{v}} M(\underline{v})$.

$\left(\varepsilon^{\prime}\right)$ A sentença existencial alternativa $\exists \underline{\underline{v}} M^{\prime}$ é uma consequência de $\mathrm{l}^{\prime}$ :

$$
\Gamma \mid \text { І. } \sqsupseteq \underline{v}\left|M(\underline{v}) \vee M_{v}\right| \text {. }
$$

$\left(v^{\prime}\right)$ A sentença universal alternativa $\left.\underline{\forall} \underline{v} M^{\prime}\right]$ é uma consequência de 1 :

$$
\Gamma \models \text { I. } \underline{\forall} \underline{v}\left[M(\underline{v}) \vee M_{v}\right] .
$$

O próximo resultado lida com o caso de um único quantificador.

Corolário 3.1. Dado um conjunto $\Gamma$ de sentenças universais, a sentença existencial $\exists v M$ é uma consequência de $\mathrm{I}$ 'se, e somente se, a sentença universal $\forall v M$ é uma consequência de $\mathrm{I}^{\prime}: \mathrm{I}^{\prime} \models_{\mathrm{L}} \exists v M$ sse $\mathrm{I}^{\prime} \models_{\mathrm{L}} \forall v M$.

Demonstração. Do teorema 3.1, temos $\mathrm{I}^{\prime} \models_{\mathrm{L}} \exists v M$ sse $\mathrm{I}^{\prime} \models_{\mathrm{L}} \forall v[M \vee M]$, e $[M \vee M]$ é logicamente equivalente a $M$. Daí, $\mathrm{I}^{\prime} \models_{\mathrm{L}} \exists v M$ sse $\mathrm{I}^{\prime} \models_{\mathrm{L}} \forall v M$.

O próximo resultado estabelece a assertiva sobre o poder das versões alternativas.

Corolário 3.2. Dada uma matriz. $M\left(v_{1}, \ldots, v_{k}, \ldots, v_{n}\right)$, seja $M_{v_{k}}^{\prime}$ sua versão alternativa $M\left(v_{1}, \ldots, v_{k}, \ldots, v_{n}\right) \vee M\left(v_{k}, \ldots, v_{k}, \ldots, v_{k}\right)$. Então, as seguintes assertivas são equivalentes para cada conjunto $\Gamma$ de sentenças universais.

( $\left.\pi^{\prime}\right)$ A sentença prenex $Q_{1} v_{1} \ldots Q_{k} v_{k} \ldots Q_{n} v_{n} M_{v_{k}}^{\prime}$ é uma consequência de I:

$$
\Gamma \models_{\text {I. }} Q_{1} v_{1} \ldots Q_{k} v_{k} \ldots Q_{n} v_{n}\left[M\left(v_{1}, \ldots, v_{k}, \ldots, v_{n}\right) \vee M\left(v_{k}, \ldots, v_{k}, \ldots, v_{k}\right)\right] .
$$

( $\left.\mu^{\prime}\right)$ A matriz alternativa $M_{v_{k}}^{\prime}$ é uma consequência de 1 :

$$
\Gamma \models_{\mathrm{I}}, M\left(v_{1}, \ldots, v_{k}, \ldots, v_{n}\right) \vee M\left(v_{k}, \ldots, v_{k}, \ldots, v_{k}\right) .
$$

Demonstração. A equivalência decorre do teorema 3.1.

$\left(\pi^{\prime}\right) \Rightarrow\left(\mu^{\prime}\right)$ Suponha I' $\models_{\mathrm{L}} Q_{1} v_{1} \ldots Q_{k} v_{k} \ldots Q_{n} v_{n} M_{v_{k}}^{\prime}$. Então I' $\models_{\mathrm{L}} \exists v_{1} \ldots \exists v_{k} \ldots \exists v_{n} M_{v_{k}}^{\prime}$ (pois $Q_{i} v_{i} F \models_{\mathrm{L}} \exists v_{i} F$ ). Assim, do teorema 3.1 resulta $\mathrm{I}^{\prime} \models_{\mathrm{L}} \forall v_{1} \ldots \forall v_{k} \ldots \forall v_{n} M_{v_{k}}^{\prime}$. Portanto, temos $\Gamma=_{\mathrm{L}} M_{v_{k}}^{\prime}$ (pois $\forall v_{i} F \models_{\mathrm{L}} F$, por aplicações de $\forall$-eliminação).

$\left(\mu^{\prime}\right) \Rightarrow\left(\pi^{\prime}\right)$ Suponha $\Gamma \models_{\mathrm{I}}, M_{v_{k}}^{\prime}$. Hintão, temos $\Gamma \models_{\mathrm{I}}, \forall v_{1} \ldots \forall v_{k} \ldots \forall v_{n} M_{v_{k}}^{\prime}$ (por aplicações de $\forall$-introdução). P'ortanto, temos $\Gamma \models_{\mathrm{I}} Q_{1} v_{1} \ldots Q_{k} v_{k} \ldots Q_{n} v_{n} M_{v_{k}}^{\prime}$ (pois $\forall v_{i} r \mid$ I. $\left.Q_{i} v_{i} F^{\prime}\right)$.

O próximo resultado estabelece a assertiva sobre uma sentença prenex e as versões singulares da sua matriz.

Corolário 3.3. Considere uma versão singular $M\left(v_{k}, \ldots, v_{k}, \ldots, v_{k}\right)$ de uma matriz $M\left(v_{1}, \ldots, v_{k}, \ldots, v_{n}\right)$. Dado um conjunto $\Gamma$ de sentenças universais, se a sentença prenex $Q_{1} v_{1} \ldots Q_{k} v_{k} \ldots Q_{n} v_{n} M\left(v_{1}, \ldots, v_{k}, \ldots, v_{n}\right)$ é uma consequência de $\Gamma$, então $a$ versão singular $M\left(v_{k}, \ldots, v_{k}, \ldots, v_{k}\right)$ também é uma consequência de $\Gamma$.

Demonstração. O argumento é similar ao anterior.

Suponha que $\Gamma \mid \mathrm{L} Q_{1} v_{1} \ldots Q_{k} v_{k} \ldots Q_{n} v_{n} M\left(v_{1}, \ldots, v_{k}, \ldots, v_{n}\right)$. Então, também tc$\operatorname{mos} \Gamma \mid \mathrm{L} \sqsupset v_{1} \ldots \sqsupset v_{k} \ldots \sqsupset v_{n} M\left(v_{1}, \ldots, v_{k}, \ldots, v_{n}\right)\left(\right.$ pois $\left.\left.Q_{i} v_{i} F^{\prime}\right|_{\mathrm{L}} F \sqsupset v_{i} F\right)$. Logo, o tcorema 3.1 dá $\Gamma \models \mathrm{L} \vee v_{1} \ldots \vee v_{k} \ldots \vee v_{n}\left[M\left(v_{1}, \ldots, v_{k}, \ldots, v_{n}\right) \vee M\left(v_{k}, \ldots, v_{k}, \ldots, v_{k}\right)\right]$. Assim, também temos $\Gamma \models_{\mathrm{L}}\left[M\left(v_{k}, \ldots, v_{k}, \ldots, v_{k}\right) \vee M\left(v_{k}, \ldots, v_{k}, \ldots, v_{k}\right)\right]$ (por $\vee$ eliminações). Portanto, $\mathrm{I}^{\prime} \models_{\mathrm{L}} M\left(v_{k}, \ldots, v_{k}, \ldots, v_{k}\right)$ (por equivalência lógica). 


\section{Caracterizações Proposicionais}

A seguir, estabeleceremos reduções proposicionais para sentenças existenciais e universais, caracterizando validades existenciais e universais como tautologias (cf. 2.2 e 2.3). Para isso, usaremos traduções que preservam conectivos ${ }^{12}$.

Como anteriormente, trabalharemos no contexto de uma teoria universal. Usaremos $\mid$ K para consequência tautológica (na linguagem proposicional K). As reduções proposicionais usam o seguinte conceito básico.

Por uma realização ${ }^{13}$ para uma linguagem $\mathrm{L}$ de primeira ordem nós entendemos um $\operatorname{par}(\mathfrak{S}, a)$ constituído por

- uma estrutura $\mathfrak{S}$ para $\mathrm{L}$, com universo $S$, e

- uma atribuição $a: \operatorname{Var} \rightarrow S$.

\subsection{Reduções proposicionais para sentenças existenciais}

Caracterizaremos agora as validades existenciais como tautologias (cf. 2.2).

Para as sentenças existenciais, empregaremos duas linguagens proposicionais similares, ambas com letras proposicionais para cada símbolo de predicado de L.

A linguagem proposicional mais simples $\mathrm{L}(v) \mathrm{tem}$ uma letra proposicional para cada símbolo de predicado de L: para cada símbolo de predicado m-ário $p$ de L, a letra proposicional correspondente $\mathrm{cm} \mathrm{L}(v)$ é $p(v, \ldots, v)$ (com $m$ v's). A outra linguagem proposicional $\mathrm{L}(v)$ estende $\mathrm{L}(v)$ pela letra proposicional adicional $v=v$.

As traduções são como se segue. Ėm ambos os casos apagamos todos os quantificadores e substituímos cada parte atômica $p\left(v_{1}, \ldots, v_{n}\right)$ por $p(v, \ldots, v)^{14}$. A diferença reside na tradução da igualdade (se presente). Cada parte atômica $x=y$ é substituída por I (verdadeiro) na tradução mais simples, e por $v=v$ na outra tradução. Desta maneira, podemos traduzir cada fórmula $F$ da linguagem de primeira ordem I para fórmulas $l_{v}(\mathrm{de} \mathrm{L}(v))$ e $l_{v}^{\prime}=(\mathrm{de} \mathrm{L}(v)=)$.

As reduções proposicionais para sentenças existenciais usam as seguintes ideias básicàs.

Considere um fragmento $\mathrm{L}^{\prime}$ da linguagem de primeira ordem $\mathrm{L}$ e uma tradução $t$ de $\mathrm{L}^{\prime}$ em uma linguagem proposicional $\mathrm{K}$. Dada a realização (S, $a$ ) para a linguagem de primeira ordem $\mathrm{L}$ e uma valoração $w$ (para as letras proposicionais) de $\mathrm{K}$, vamos chamá-las de associadas sob $t$ se, e somente se, para cada fórmula atômica $A$ de $\mathrm{L}^{\prime}$, com tradução $A^{t}$ (em K), temos $\mathfrak{S} \models A \llbracket a \rrbracket$ sse $w\left[A^{t}\right]=1$.

O próximo resultado é imediato.

Lema 4.1. Dada uma tradução t de $\mathrm{L}^{\prime}$ em $\mathrm{K}$, como acima, considere uma realização $(\mathfrak{S}, a)$ para $\mathrm{L}$ e uma valoração w para $\mathrm{L}_{l}$ que sejam associadas sob t. Então, para toda matriz $F$ em $\mathrm{L}^{\prime}$ com tradução $F^{t}$ em $\mathrm{L}_{t}$, temos $\mathcal{S} \vdash F \| a \rrbracket$ se, e somente se, $w\left[F^{t}\right\rceil-1$.

Demonstração. Por indução: a base provém da hipótese de associação.

Agora, podemos caracterizar os teoremas existenciais em termos de consequências tautológicas. 
Teorema 4.1. Dado um conjunto l' de sentenças universais e uma matriz. $M$ em $\mathrm{L}$, considere suas traduções em $\mathrm{L}(v)$ e $\mathrm{L}(v)$ como dadas acima. Então, as seguintes assertivas são equivalentes.

(ع) A sentença existencial $\exists v_{1} \ldots \exists v_{n}$ Mé uma consequência de $\Gamma$ : $\Gamma=_{\mathrm{L}} \exists v_{1} \ldots \exists v_{n} M$.

(.v) A versão singular proposicional $M_{v}$ é uma consequência tautológica do conjunto $\Gamma_{v}: \Gamma_{v}=\mathrm{L}_{(v)} M_{v}$.

$\left(_{v}\right)$ A versão singular proposicional $M_{v}$ é uma consequência tautológica do conjunto $\Gamma_{v} \cup\{v=v\}: \Gamma_{v} \cup\{v=v\} \models \mathrm{L}_{(v)-} M_{v}$.

Demonstração. Mostraremos $(. v) \Rightarrow(\varepsilon) \Rightarrow(. \bar{v}) \Rightarrow(. v)$.

$(. v)>(\varepsilon)$ Suponha que não $t c m o s(\varepsilon): \Gamma \chi_{\mathrm{I}} . \sqsupset v_{1} \ldots \sqsupset v_{n} M$. Então, para algum modelo

S | $\Gamma$ c alguma atribuição $a:$ Var $>S$, temos $5 X M\|a\|$. Defina a valoração $w$ para $\mathrm{L}_{v}^{=}$por $w\left|\Lambda_{v}\right| \quad 1 \operatorname{ssc}\left(5 \mid \quad \Lambda\|a\|\right.$. Desse modo, $w$ é uma valoração para $\mathrm{L}_{v}^{=}$associada a $(\mathfrak{S}, a)$. Assim, o lema 4.1 nos dá:

(i) $w\left[G_{v}\right]=1$, para cada sentença $\forall x_{1} \ldots \forall x_{m} G$ em $\Gamma$ (pois, $\mathcal{S} \models A[[a]$ );

(ii) $w\left[M_{v}\right] \neq 1$ (pois $\left.\mathfrak{S} \not \models M \llbracket[a]\right]$ ).

Portanto, a valoração $w$ satisfaz cada fórmula de $\Gamma_{v}$, mas não satisfaz. $M_{v}$.

$(\varepsilon) \Rightarrow(\cdot v)$. Suponha que não temos $\left(._{v}\right): \Gamma_{v} \not \not_{L_{v}} M_{v}^{=}$. Hintão, alguma valoração $w$ para $\mathrm{L}(v)=$ satisfar. $\Gamma_{v}^{=}$mas não $M_{v}^{=}$. I)efina uma estrutura $S$ para $\mathrm{L}$. com um único elemento (c universo $\{s\})$ estipulando $\langle s, \ldots, s\rangle \subset p^{\mathbb{S}} \operatorname{ssc} w|p(v, \cdots, v)| \quad$ 1. Considerando a atribuição constantc $a: \operatorname{Var}>S(\operatorname{com} a(v): s), \operatorname{vemos}$ que a realização $(\mathfrak{5}, a)$ é associada à valoração $w$ de $\mathrm{L}(v)$. Assim, o lema 4.1 nos dá:

1. $\mathfrak{S} \models G\left[[a]\right.$, para cada sentença $\forall x_{1} \ldots \forall x_{m} G$ em I' (pois $w\left[G_{v}\right]=1$ );

2. $5 \times M\|a\|\left(\right.$ pois $\left.w\left|M_{v}^{=}\right| \quad 0\right)$.

Logo, a estrutura S é um modelo (com um único elemento) de l' que não satisfaz a sentença $\exists v_{1} \ldots \exists v_{n} M$.

$\left(._{v}\right) \Rightarrow\left({ }_{v}\right)$. Suponha $\Gamma_{v}^{=} \models_{\mathrm{I}_{(v)^{-}}} M_{v}^{=}$. I)ada uma valoração $w$ para $\mathrm{L}(v)$ que satisfaz. $\Gamma_{v}$, cstenda-a para $\mathrm{L}_{v}^{=}$fazendo $w|v \quad v|: \quad 1$. A valoração $\mathrm{L}_{v}^{=}$estendida satisfará $\Gamma_{v}, \mathrm{e}$, portanto, também $M_{v}^{=}$. Nessc caso, a valoração inicial $w$ de $\mathrm{L}(v)$ satisfará $M_{v}{ }^{15}$.

\subsection{Reduções proposicionais para sentenças universais}

Caracterizaremos agora as validades universais como tautologias (cf. 2.3).

Para as sentenças universais, podemos empregar uma linguagem proposicional com uma letra proposicional para cada fórmula atômica de L. Contudo, é mais conveniente proceder da maneira seguinte.

Considere um conjunto $\underline{v}:=\left\{v_{1}, \ldots, v_{n}\right\}$ de variáveis em L. Usaremos I. $[\underline{v}]$ para o fragmento $\underline{v}$ de L composto das fórmulas com variáveis livres no conjunto $\underline{v}$ dado. Agora, consideramos a linguagem proposicional $\mathrm{L}(\underline{v})$ com as letras $p\left(v_{i_{1}}, \ldots, v_{i_{m}}\right)$ : uma 
Ietra para cada fórmula atômicá de $\mathrm{L}|\underline{v}|$ (incluindo as igualdades $v_{i_{j}} \quad v_{i_{k}}$, quando $\mathrm{L}$ possui o símbolo de igualdade).

A tradução é feila simplesmente apagando-sc os quantificadores. Desse modo, podemos traduzir cada fórmula $F$ do fragmento $\mathrm{L}[\underline{v}]$ de $\mathrm{L}$ a uma fórmula $F_{\underline{v}} \mathrm{de} \mathrm{L}(\underline{v})$.

Considere uma substituição de variáveis $h: \operatorname{Var} \rightarrow$ Var. Dada uma matriz $F$, usamos $F^{h}$ para o resultado da aplicação de $h$ a cla, ou seja $F\left(x_{1}, \ldots, x_{m}\right)^{h}:=F\left(h\left(x_{1}\right), \ldots, h\left(x_{m}\right)\right)$. Agora, dada uma sentença universal $G$, digamos $\forall x_{1} \ldots \forall x_{m} F$, considere sua matriz $F$, e forme o conjunto $G^{v}$ de todas as suas instâncias $\underline{v}$, i.e. $G^{v}:=\left\{F^{h}: h:\right.$ Var $\left.\rightarrow \underline{v}\right\}$. Para um conjunto $\Gamma$ de sentenças universais, $\Gamma \underline{v}$ é definido como esperado: consiste de todas as instâncias $\underline{v}$ das matrizes de suas sentenças.

Note que os axiomas para a identidade (equivalências e congruências ${ }^{16}$ ) são sentenças universais. Usaremos $J$ para o conjunto de axiomas para a identidade e $J \underline{v}$ para o conjunto de todas as suas instâncias $\underline{v}$.

As reduções para sentenças universais usarão as seguintes idéias auxiliares.

Temos uma construção canônica (sem recorrer a testemunhas). Dado um conjunto $\Delta$ de fórmulas de $\mathrm{L}(\underline{v})$, definimos a estrutura canônica $\mathfrak{D}$ (bascada $\mathrm{cm} \Delta$ ) basicamente como usual ${ }^{17}$. Primeiramente, definimos uma relação binária no conjunto $\underline{v}$ de variáveis por $v_{i_{j}} \sim v_{i_{k}}$ sse $\Delta \vdash v_{i_{j}}=v_{i_{k}}{ }^{18}$. Agora, tomamos o universo como o quociente: $D:=\underline{v} / \sim$. Assim, temos uma projeção natural $q: \underline{v} \rightarrow D$ dada por $q(v):=v / \sim$. Finalmente, definimos $p^{\mathfrak{D}}:=\left\{\left\langle v_{i_{1}} / \sim, \ldots, v_{i_{m}} / \sim\right\rangle: \Delta \vdash p\left(v_{i_{1}}, \ldots, v_{i_{m}}\right)\right\}^{19}$.

Dada uma substituição $h: \operatorname{Var} \rightarrow \underline{v}$, a composta $q \circ h(\operatorname{com}(q \circ h)(v):=q(h(v)))$ é uma atribuição $q \circ h:$ Var $\rightarrow D$ de variáveis no universo $D$. Conversamente, cada atribuição $a$ : Var $>D$ ć da forma $a \quad q \circ h$, para alguma substituição de variáveis $h:$ Var $>\underline{v}^{20}$.

O próximo resultado estabelece a propriedade familiar da estrutura canônica baseada $\mathrm{em}$ um conjunto (proposicionalmente) maximal consistente $D^{21}$.

Lema 4.2. Dado um conjunto $\Delta$ de fórmulas em $\mathrm{L}(\underline{v})$ que é (proposicionalmente) maximal consistente, considere a estrutura canônica $\mathfrak{D}$ baseada em $\Delta$. Entāo, para cada matriz $\mathrm{F}$ em $\mathrm{L}|\underline{v}|$ e cada substituição de variáveis $h:$ Var $\quad$ y, temos que $\mathfrak{D} \mid \quad r\|q \circ h\|$ se, e somente se, $F^{\text {h }} \subset \Delta$.

Demonstração. Por indução: a base provém da construção e o passo indutivo provém da hipótese indutiva e da consistência máximal ${ }^{22}$.

Agora, podemos caracterizar os teoremas universais em termos de consequências tautológicas.

Teorema 4.2. Dado um conjunto $\Gamma$ de sentenças universais de $\mathrm{I},[\underline{v}]$, Considere os

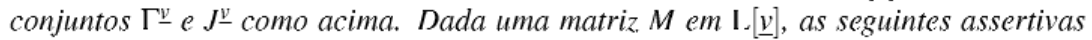
são equivalentes.

(v) $\Lambda$ sentença universal $\forall v_{1} \ldots \forall v_{n} M$ é uma consequência de $\Gamma$ : $\Gamma \mid$ I. $\forall v_{1} \ldots \forall v_{n} M$.

$\left(v_{n}\right)$ A sentença universal $\forall v_{1} \ldots \forall v_{n} M$ é satisfeita em todo modelo de $\mathrm{I}$ com, no máximo, n elementos.

$(\cdot \underline{v}) \Lambda$ matriz $M$ é uma consequência tautológica de $\Gamma^{v} \cup . J^{v}: \Gamma^{v} \cup J^{v} \mid \mathrm{I}_{(v)} M$. 
Demonstração. Claramente $(v) \Rightarrow\left(v_{n}\right)$. Mostraremos $\left(v_{n}\right) \Rightarrow\left({ }_{\cdot v}\right)$ e $(\cdot v) \Rightarrow(v)$.

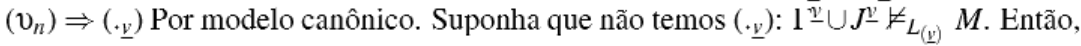
a matriz. $M$ não é uma consequência tautológica de $\Gamma^{\underline{v}} \cup J \underline{J}$. Assim, $\Gamma^{\underline{v}} \cup J \underline{v} \cup\{\neg M\}$ é proposicionalmente consistente $(\mathrm{cm} \mathrm{L}(\underline{v}))$. Estenda esse conjunto a um conjunto (proposicionalmente) máximal consistente $\Delta(\mathrm{cm} \mathrm{L}(\underline{v}))$ e considere a cstrutura canônica $\mathfrak{D}$ bascada $\mathrm{em} \Delta$. Notc que, pelo lema 4.2 , temos:

(i) a estrutura $\mathfrak{D}$ é um modelo de $\mathrm{I}^{\prime}(\mathfrak{D} \models 1$ ');

(ii) a cstrutura $\mathfrak{D}$ não é um modelo de $\forall v_{1} \ldots \forall v_{n} M$ (D $\left.\bigvee \forall v_{1} \ldots \forall v_{n} M\right)$.

Podemos ver essas duas alegações como se segue.

(i) Considere uma sentença universal $\forall x_{1} \ldots \forall x_{n} F \mathrm{~cm} \Gamma$. Iada uma atribuição de variáveis $a: \operatorname{Var} \rightarrow D$, temos $a=q \circ h$, para alguma substituição de variáveis $h:$ Var $\rightarrow \underline{v}$. Ora, como $F^{h}$ está no subconjunto $\Gamma^{\underline{v}} \subseteq \Delta$, o lemma 4.2 nos dá $\mathfrak{D} \models F[[q \circ h]]$, ou scja, $\mathfrak{D} \models F[[a]]$.

(ii) Note que a matriz $\neg M$ está em $\Delta$ e considere a substituição identidade $i$ (dada por $i(v): \quad v)$. Pelo lemma 4.2, $\mathfrak{D} \mid \neg M\|q \circ i\|$, c assim $\mathfrak{D} X M\|q\|$, donde obtemos D $X \forall v_{1} \ldots \forall v_{n} M$.

Desse modo, temos um modelo $\mathfrak{D}$ de $\mathrm{l}$, com no máximo $n$ elementos, que não satisfaz a sentença universal $\forall v_{1} \ldots \forall v_{n} M$.

$(. v) \Rightarrow(v)$ Temos uma derivação (em $\mathrm{L}(\underline{v})$ ) de $M$ a partir de subconjuntos $\Gamma^{\prime} \subseteq \Gamma^{\underline{v}}$ e $J^{\prime} \subseteq J \underline{v}$. Agora, basta notar que:

1. de $\Gamma$, podemos obter as instâncias $\mathrm{cm} \Gamma^{\prime}$ por $\vee$-climinações;

2. dos axiomas em $J$ resultam as instâncias em $J^{\prime}$, também por $\forall$-eliminações.

Assim, temos uma derivação (cm L), a partir de $\Gamma$, da matriz $M$, logo também da sentença universal $\vee v_{1} \ldots \vee v_{n} M$ (por $\vee$-introduções). Portanto, $\Gamma=_{\mathrm{L}} \vee v_{1} \ldots \vee v_{n} M$.

Note que a equivalência $(v) \Leftrightarrow\left(v_{n}\right)$ estabelece a condição de validade para sentenças universais mencionada na seção 3 acima.

\section{Retrospecto: conceitos e resultados}

Agora, vamos repassar a situação, resumindo nossos conceitos e resultados principais.

Tratamos de três problemas (relacionados):

1. o problema das validades existenciais (cf. 2.1 e 3);

2. caracterizações proposicionais para sentenças existenciais como tautologias (cf. 2.2 e 4.1);

3. caracterizações proposicionais para sentenças universais como tautologias (cf. 2.3 e 4.2 ). 
O primeiro problema refere-se à questão das validades existenciais; os outros dois são relacionados a este e fornecem algumas clarificações interessantes.

Trabalhamos no contexto de uma teoria universal. Dada uma linguagem L de primeira ordem, sem símbolos para constantes ou funções, consideramos um conjunto $\Gamma$ de sentenças universais de $\mathrm{L}$.

\subsection{Consequências existenciais e universais}

Inicialmente, vamos repassar consequências existenciais e universais (cf. seção 3).

Introduzimos duas transformações são sintáticas simples e relacionadas. Elas se baseiam em uma fórmula de L e uma de suas variáveis.

\begin{tabular}{c|cc} 
Fórmula $F$ & Instância singular $F_{v_{k}}$ & Versão alternativa $F_{v_{k}}^{\prime}$ \\
\hline$F\left(v_{1}, \ldots, v_{k}, \ldots, v_{n}\right)$ & $F\left(v_{k}, \ldots, v_{k}, \ldots, v_{k}\right)$ & $F\left(v_{1}, \ldots, v_{k}, \ldots, v_{n}\right) \vee F\left(v_{k}, \ldots, v_{k}, \ldots, v_{k}\right)$
\end{tabular}

O lema 3.1 dá a a equivalência entre fechos existenciais e suas versões alternativas

A sentença existencialmente quantificada $\exists \underline{v} F$ é logicamente equivalente a sua versão alternativa $\underline{\varrho} \underline{v}\left|F \vee F_{v}\right|(\operatorname{com} v \mathrm{~cm} \underline{v})$ :

$$
\exists \underline{v} F \vdash \underline{v} F_{v}^{\prime} \mathrm{c} \exists \underline{v} F_{v}^{\prime} \vdash \exists \underline{v} F \text {. }
$$

Como estamos interessados em saber se uma sentença existencial $\sqsupseteq \underline{v} M$ é consequência de $\Gamma$, podemos muito bem substituí-la por qualquer outra equivalente, em particular por essa transformada sintática simples.

Além disso, estabelecemos algumas conexões entre consequências existenciais e universais (cf. proposição 3.1 e teorema 3.1). Dada uma matriz $M$ de L, com conjunto $\underline{v}$ de variáveis e uma variável $v \in \underline{v}$, considere as sentenças: seu fecho existencial $\exists \underline{v} M$, sentença existencial alternativa $\underline{\exists} \underline{v} M_{v}^{\prime}$ e sentença universal alternativa $\underline{\forall} \underline{v} M_{v}^{\prime}$.

( $\varepsilon)$

$$
\text { A sentença existencial } \exists v M \text { é uma consequência de } \mathrm{I}^{\prime} \quad \mathrm{I}^{\prime} \models_{\mathrm{L}} \exists v M
$$

步

$\left(\varepsilon_{1}\right) \quad$ A sentença existencial $\exists v M$ vale em todo modelo de $\Gamma \quad$ com um único elemento $\Uparrow$

$\left(\varepsilon^{\prime}\right) \quad$ A sentença cxistencial alternativa $\exists \underline{v} \underline{M_{v}^{\prime}}$ é consequência de $\Gamma \quad \Gamma=_{\mathrm{L}} \exists \underline{v} \underline{ }\left[M \vee M_{v}\right]$ 代

(v') A sentença universal alternativa $\underline{\forall} \underline{v} M_{v}^{\prime}$ é consequência de $\Gamma \quad \Gamma|\mathrm{L} \underline{\forall} \underline{v}| M \vee M_{v} \mid$

$\mathrm{O}$ caso de um de um único quantificador é bastante simples (cf. corolário 3.1).

(ع) A sentença existencial $\exists v M$ é consequência de $\Gamma \quad\left(\Gamma \models_{\mathrm{I}} . \exists v M\right)$

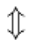

(v) A sentença universal $\forall v M$ é consequência de $\Gamma \quad$ ( $\Gamma \mid$ I. $\forall v M)$

Temos uma conexão entre versões alternativas (cf. corolário 3.2).

$\left(\pi^{\prime}\right) \quad$ A sentença prenex $\underline{Q} \underline{v}\left[M \vee M_{v}\right]$ é consequência de $\Gamma \quad\left(\Gamma \models_{\mathrm{L}} \underline{Q} \underline{v} \underline{ }\left[M \vee M_{v}\right]\right)$ $\Uparrow$

(v) A matriz alternativa $\left[M \vee M_{v}\right]$ é consequência de $\Gamma \quad\left(\Gamma \models_{\mathrm{L}}\left[M \vee M_{v}\right]\right)$

doispontos, Curitiba, São Carlos, vol. 6, n. 2, p.145-163, outubro, 2009 
Temos também a seguinte conexão entre uma sentença prenex e as versões singulares da sua matriz (cf. corolário 3.3).

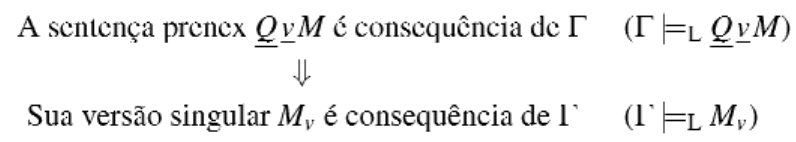

\subsection{Caracterizações proposicionais para sentenças existenciais}

Agora, vamos resumir nossas reduções proposicionais para sentenças existenciais (cf 4.1).

Para sentenças existenciais, utilizamos duas linguagens proposicionais similares, tendo letras proposicionais correspondendo aos (símbolos de) predicados de L.

1. Linguagem proposicional $\mathrm{L}(v)$ :

$$
\text { predicado } \mathrm{m} \text {-ário de } \mathrm{L} \mapsto \text { letra proposicional } p(v, \ldots, v)(\mathrm{m} v \text { 's }) \text {. }
$$

2. L inguagem proposicional $\mathrm{L}(v)=:=\mathrm{L}(v) \cup\{v=v\}$.

Traduções $\mathrm{em} \mathrm{L}(v) \mathrm{e} \mathrm{L}(v)$, apagar os quantificadores $\mathrm{e}$ traduzir as partes atômicas:

\begin{tabular}{c|ccc} 
predicado & igualdade \\
\hline$p\left(v_{1}, \ldots, v_{n}\right) \mapsto p(v, \ldots, v)$ & $(x=y)_{v}:=T^{\top} \quad(x=y)_{v} \quad:=v=v$
\end{tabular}

Temos a seguinte caracterização para os teoremas existenciais em termos de consequências tautológicas (cீ. teorema 4.1).

(c) A sentença existencial $\sqsupset v_{1} \ldots \sqsupset v_{n} M$ é consequência de $\Gamma \quad \Gamma \mid$ ı. $\sqsupset v_{1} \ldots \sqsupset v_{n} M$ $\Uparrow$

(.v) A tradução singular $M_{v}$ é consequência tautológica de $\left.\Gamma_{v} \quad \Gamma_{v}\right|_{\mathrm{I}_{(v)}} M_{v}$ $\Uparrow$

$\left({ }_{v}\right) \quad$ A tradução $M_{v}$ é consequência tautológica de $\Gamma_{v} \cup\{v=v\} \quad \Gamma_{v} \cup\{v=v\}=\mathrm{L}_{(v)}$.

\subsection{Caracterizações proposicionais para sentenças universais}

Agora, vamos resumir nossas reduções proposicionais para sentenças universais (cf 4.1).

Para as sentenças universais, consideramos o fragmento $L[\underline{y}\rfloor$ de $\mathrm{L}$. composto das fórmulas com variáveis livres num conjunto dado $\underline{v}:=\left\{v_{1}, \ldots, v_{n}\right\}$ de variáveis.

I inguagem proposicional L $(\underline{v})$ :

$$
\begin{array}{ccc}
\left(\begin{array}{c}
\text { predicado m-ário de } \mathrm{L} \\
\text { variáveis } v_{i_{1}}, \ldots, v_{i_{m}} \in \underline{v}
\end{array}\right) & \mapsto \begin{array}{l}
\text { letra proposicional } p\left(v_{i_{1}}, \ldots, v_{i_{m}}\right) \\
\text { variáveis } v_{i_{j}}, v_{i_{k}} \in \underline{v}
\end{array} & \mapsto \text { letra proposicional } v_{i_{j}}=v_{i_{k}}
\end{array}
$$


Fistrutura canônica $\mathfrak{D}$ baseada em conjunto $\Delta$ de fórmulas de $\mathrm{L}(\underline{\underline{v}})$

1. Relação binária sobre $\underline{v}: v_{i_{j}} \sim v_{i_{k}} \operatorname{ssc} \Delta \vdash v_{i_{j}}=v_{i_{k}}$.

2. Universo $D:=\underline{v} / \sim$, com projeção natural $q: \underline{v} \rightarrow D$.

3. Predicados: $p^{\mathfrak{D}}:=\left\{\left\langle v_{i_{1}} / \sim, \ldots, v_{i_{m}} / \sim\right\rangle: \Delta \vdash p\left(v_{i_{1}}, \ldots, v_{i_{m}}\right)\right\}$.

Propricdade da estrutura canônica $\mathfrak{D}$ bascada em conjunto $\Delta$ maximal consistente $\mathrm{cm} \mathrm{L}(\underline{v})$ (cf. lema 4.2). Para matriz $F \mathrm{~cm} \mathrm{~L}|\underline{v}| \mathrm{c}$ substituição $h:$ Var $>\underline{v}$ :

$$
\mathfrak{D} \models F[[q \circ h]] \text { sse } F^{h} \in \Delta .
$$

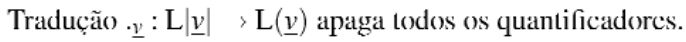

Temos a seguinte caracterização para os teoremas universais $\mathrm{cm}$ termos de consequências tautológicas (cl. teorema 4.2).
(v) A sentença universal $\forall v_{1} \ldots \forall v_{n} M$ é uma consequência de $\Gamma \quad \Gamma \mid{ }_{\mathrm{L}} \forall v_{1} \ldots \forall v_{n} M$
$\Uparrow$
$\left(v_{n}\right) \quad$ A sentença universal $\forall v_{1} \ldots \forall v_{n} M$ vale cm todo modelo de $\Gamma \quad$ com, no máximo, $n$ elementos $\Uparrow$
$(\cdot \underline{v}) \quad$ A matriz $M$ é uma consequência tautológica de $\Gamma^{\underline{v}} \cup J^{\underline{v}} \quad \Gamma^{\underline{v}} \cup J^{\underline{v}} \mid \mathrm{L}_{(v)} M$

\section{Conclusão}

Agora, vamos tecer alguns comentários possíveis desdobramentos e extensões de nossos resultados, colocando-os em contexto.

\subsection{Possíveis extensões}

Apresentaremos alguns possíveis desdobramentos e extensões de nossos resultados.

Não parece difcil estender nosso enfoque a linguagens com símbolos para constantes mas não para funções.

Vejamos alguns exemplos e ideias sugeridas por eles.

1. Com um único símbolo de constante $c$.

(a) A sentença existencial $\exists x x=c$ é válida, sem que sua versão universal $\forall x x=$ $c$ seja válida. Mas, $\exists x x=c$ é logicamente equivalente a $\exists x(x=c \vee c=c) \mathrm{e}$ a versão universal desta, $\forall x(x=c \vee c=c)$, também é válida.

(b) A sentença existencial $\exists x \neg x=c$ não é válida, sendo logicamente equivalente a $\exists x(\neg x=c \vee \neg c=c)$ e a versão universal desta, $\forall x(\neg x=c \vee \neg c=c)$, tampouco é válida. 
2. Com dois símbolos de constantes $c$ ed.

(a) A sentença existencial $\exists x(x=c \vee x=d)$ é válida, sem que sua versão universal $\forall x(x=c \vee x=d)$ seja válida. Mas, $\exists x(x=c \vee x=d)$ é logicamente equivalente a $\exists x[(x=c \vee x=d) \vee(c=c \vee c=d)) \vee(d=c \vee d=d)]$ e a versão universal desta, $\forall x[(x=c \vee x=d) \vee(c=c \vee c=d)) \vee(d=c \vee d=d)]$, também é válida.

(b) A sentença existencial $\exists x(x=c \wedge x=d)$ não é válida, sendo logicamente equivalente a $\exists x[(x=c \wedge x=d) \vee(c=c \wedge c=d) \vee(d=c \wedge d=d)] \mathrm{c}$ a versão universal desta, $\forall x[(x=c \wedge x=d) \vee(c=c \wedge c=d) \vee(d=c \wedge d=d)]$, tampouco é válida.

(c) Não podemos mais usar instâncias singulares (nem mesmo para sentenças scm quantificadores): a matriz $c=d$ não é válida, mas é satisfeita $\mathrm{cm}$ toda estrutura com um único elemento.

Agora, a transformação sintática envolve substituições pelas constantes. No caso de dois símbolos de constantes $c$ e $d$, A sentença existencial $\sqsupset x q(x)$ será transformada $\mathrm{cm} \sqsupset x|q(x) \vee q(c) \vee q(d)|$. A transformadada da sentença cxistencial $\sqsupset x \sqsupset y r(x, y)$ terá como matriz uma disjunção, cada disjunto sendo a matriz original $r(x, y)$ ou obtido dela por uma substiuiação das variáveis originais $x$ e $y$ pelas constantes $c \mathrm{e} d$ :

$$
r(x, y), r(c, c), r(c, d), r(d, x), r(d, c), r(d, d) .
$$

As reduões proposicionais também parecem poder ser estendidas.

Essas ideias são sugeridas pelas seguinte generalizações razoáveis das condições de validade mencionadas na seção 3. Considere uma matriz $M\left(v_{1}, \ldots, v_{n}\right)$ com no máximo $k>0$ símbolos para constantes.

( $\left.\varepsilon_{k}\right)$ A sentença existencial $\exists v_{1} \ldots \exists v_{n} M\left(v_{1}, \ldots, v_{n}\right)$ é válida sse cla vale toda estrutura com, no máximo, $k$ elementos.

$\left(v_{n \mid k}\right)$ A sentença universal $\forall v_{1} \ldots \forall v_{n} M\left(v_{1}, \ldots, v_{n}\right)$ é válida sse ela vale toda estrutura com, no máximo, $n+k$ elementos.

Assim, a estensão de nossos resultados a linguagens com símbolos para constantes parece faclível. O caso de linguagens com símbolos para funções parece bem mais problemático. Parte da dificuldade deve-se à ausência de colas superiores como acima: há sentenças existenciais satisfeitas em todas as estruturas finitas que não são válidas. Um exemplo é $\exists x \exists y \exists z,[f(x)=f(y) \rightarrow f(z)=c]^{23}$.

\subsection{Comentários finais}

Agora, vamos fazer alguns comentários finais sobre nosso enfoque, colocando-o em contexto.

Estávamos interessados em sentenças existenciais que são logicamente válidas. Nossos resultados estão no contexto de uma teoria universal. Portanto, eles são claramente aplicáveis ao caso que estamos interessados: a teoria $\varnothing$. 
Parece ser interessante comparar nosso enfoque com um resultado conhecido: o chamado Teorema de Consistência de Hilbert e Ackermann [Sho67, p. 49], que fornece reduções proposicionais para a consistência de um conjunto de sentenças universais. Esse resultado também fornece uma caracterização das sentenças existenciais que são consequências de um conjunto $\Gamma$ de sentenças universais:

Uma sentença existencial $\underline{\exists} \underline{v} M$ é consequência de um conjunto $\Gamma$ de sentenças universais se, e somente se, existe uma disjunção de instâncias da matriz $M$ que seja consequência de $\Gamma$.

Vamos comparar essa caracterização com nossos resultados (cf. teorema 3.1).

1. Por um lado, essa caracterização é mais geral do que nossos resultados, uma vez que ela se aplica a linguagens de primeira ordem que podem ter (símbolos para) constantes e funções.

2. Por outro lado, essa caracterização meramente assegura a existência de alguma disjunção de instâncias da matriz, enquanto que nossos resultados explicitam uma disjunção para cada caso.

Por cxcmplo, considere a questão: quando $\Gamma \mid \sqsupset x \sqsupset y \sqsupset z M(x, y, z)$ ?

- A caracterização acima daria quando alguma disjunção $M\left(u_{1}, v_{1}, w_{1}\right) \vee \ldots \vee M\left(u_{j}, v_{j}, w_{j}\right)$ é consequência de $\Gamma$.

- Nossos resultados dão quando $M(x, y, z) \vee M(y, y, y)$ é consequência de $\Gamma$.

Nesse sentido, nosso enfoque, ainda que restrito, sendo construtivo, é mais informativo.

1 ver, por exemplo, "Hume's Dialogue on Natural Religion” (Part IX, 189)[Hum92], a Introdução da Lógica de Kant (Jäsche)[Kan92], Orenstein[Ore73] p. 62 e Quine[Qui54].

2 Note que uma definição de "pintalouva" ou outras elucidações não são de muita ajuda. Primeiro, elas seriam contadas como hipóteses. Segundo, se alguém explica "pintalouva" como, digamos, "aquelas coisas que são minsicais”, estamos introduzindo “minsicais” no cenário, e voltamos para a mesma questão. 
3 Uma assertiva como "Pégaso é um cavalo alado" pode ser parafraseada como “algo idêntico a Pégaso é um cavalo alado".

${ }^{4}$ Esses compromissos são incorporados na regra de introdução do $\exists$ em Dedução Natural.

5 Esse compromisso é que permite inferir $\exists v F$ de $\forall v F$.

${ }^{6}$ Em termos intuitivos, a idéia pode ser apresentada da seguinte maneira. Considere uma estrutura com um único elemento, com universo $\{s\}$. Para cada $n$, nós temos uma única nupla, nomeadamente $<s, \ldots, s>$. Ou seja, a informação que a estrutura fornece é se esta n-upla está numa relação n-ária ou não.

${ }^{7}$ Alternativamente, pode-se usar uma constante $c$.

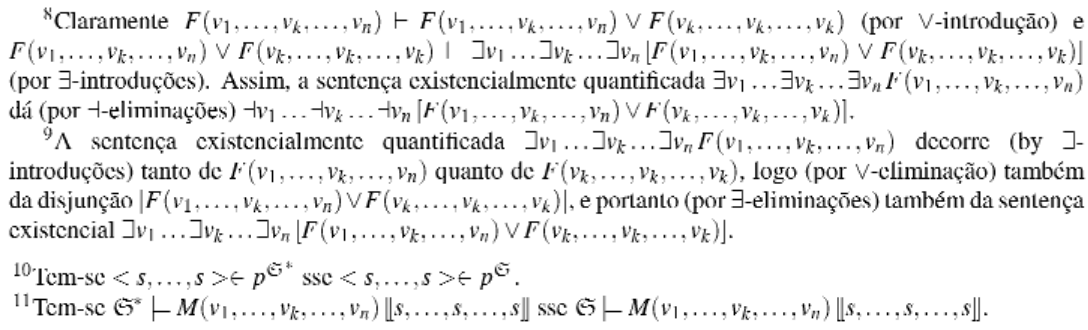

${ }^{12}$ Os métodos a serem empregados aqui sāo similares a algumas ideias encontradas na literatura [Cha79, Lind72, Men66, Sho67].

${ }^{13}$ Fste conceito também é chamado 'interpretação' [FFT84, p. 27].

${ }^{14}$ Tais traduções singulares aparecem na literatura, p. ex. "réduction de genre un" |Cha79, p. 119-123| e [Men66, p. 59]. Flas costumam ser usadas para estabelecer a consistência relativa da lógica de primeira ordem com respeito à lógica proposicional: mostra-se que a traduçāo é uma interpretaçāo transformando tcoremas cm tcoremas. $\Lambda$ qui, mostraremos que tais interpretações são fićis para as sentenças cxistenciais.

${ }^{15}$ Alternativamente,podemos notar que a tradução natural de $\mathrm{I}(v){ }^{-}$em $\mathrm{I} .(v)$ (com identidade em todos os casos, salvo $v=v+$, é uma interpretaçāo: ela mapeia axiomas em axiomas e regras em regras.

${ }^{16}$ ()s axiomas de conguência, para cada símbolo de predicado m-ário $p$, são os fechos universais das fórmulas $\left[p\left(x_{1}, \ldots, x_{j}, \ldots, x_{m}\right) \wedge x_{j} \quad y_{j} \rightarrow p\left(x_{1}, \ldots, y_{j}, \ldots, x_{m}\right)\right]$.

${ }^{17} \mathrm{~A}$ construçāo mais comum parte de um conjunto de fórmulas de primeira ordem. Aqui, em contraste, partircmos de um conjunto de fórmulas proposicionais.

${ }^{1 *}$ Note que $\sim$ é uma relaçāo de equivalência no conjunto de variáveis $\underline{v}$.

${ }^{19}$ Note que $p^{\mathfrak{D}}$ está bem definida devido aos axiomas para a identidade.

${ }^{201}$ De fato, para cada $v \subset$ Var, selecione uma variável $u_{v}$ na classe $a(v)$ e defina $h(v):=u_{v}$; entāo temos $q(h(v))-u_{v} / \sim-a(v)$.

${ }^{21}$ Note que, neste caso, $\Delta \vdash F$ sse $F \subset \Delta$, e, $F \not \subset \Delta$ sse $\neg F \subset \Delta$.

${ }^{22}$ Por exemplo, $\mathfrak{D} \mid \quad F \llbracket q \circ h \rrbracket \mathrm{sse}$ (pela definição de satisfação) $\mathfrak{D} K F \llbracket q \circ h \rrbracket \mathrm{sse}$ (pela hipótese indutiva) $F^{h} \notin \wedge$ sse (pela consistência máximal) $(\neg F)^{h} \in \Lambda$.

${ }^{23}$ De fato, em uma estrutura finita $\mathfrak{F}, f^{\mathfrak{s}}$ sendo injetiva, será sobrejetiva; mas ela nāo vale nos naturais com succssor como $f^{\Re} \mathrm{c} c^{\Re}:-0$.

\section{Referências bibliográficas}

[Cha79] Chauvineau Jean. La logique moderne. Que sais-je? Presses

Universitaires de France, Paris, 2e edition, 1979. 
[EFT84] H. D. Ebbinghaus, J. Flum \& W. Thomas. Mathematical Logic. Springer-Verlag, Berlim, 1984.

[End72] Herbert B. Enderton. A Mathematical Introduction to Logic. Academic Press, New York, 1972.

[Hum92] David Hume. Diálogos sobre a Religião Natural. Martins Fontes, 1992.

[Kan92] Immanuel Kant. Lógica Biblioteca Tempo Universitário, 93, Tempo Brasileiro, Rio de janeiro, 1992.

[Men66] Elliot Mendelson. Introduction to Mathematical Logic. D. van Nostrand, Princeton, 1966.

[Ore73] Alex Orenstein. On Explicating Existence in Terms of Quantification, in Logic and Ontology, ed. Milton K. Munitz, NYU Press, New York, 1973.

[Qui54] W.V. Quine. Quantification in the Empty Domain, in The Journal of Symbolic Logic, volume 19, no.3,1954, pp.177--179

[Sho67] Joseph R. Shoenfield. Mathematical Logic. Addison-Wesley, Reading, 1967.

[vDa89] Dirk van Dalen. Logic and Structure. Springer-Verlag, Berlim, 2nd edition, 1989. 